may well offer a solution to users of contacts in highly sensitive circuits.

This work has demonstrated the usefulness of ellipsometry as a technique for studying the cleanliness of light duty noble metal contact surfaces.

However, as indicated earlier, ellipsometric techniques have much to offer in a wide variety of fields. Other studies are continuing on ion adsorption on gold in various environments with the object of throwing light on such important technological problems as environmental control and catalysis, and further studies on several aesthetic aspects of commercial gold surfaces have been initiated.

Ellipsometry does not provide all the answers to surface problems and it is usually best operated in conjunction with other techniques measuring important experimental parameters.

Acknowledgements are due to the Science Research Council under whose funding the ellipsometer was constructed and now operates.

\title{
Control of Particle Size in Gold Suspensions
}

Monodisperse suspensions of gold particles are often used in the study of particle size-dependent phenomena such as Brownian motion, light scattering, sedimentation and electrophoresis of small particles. A monodisperse system is a colloidal suspension in which all the particles are of effectively the same size. In many applications gold suspensions have the advantage of being relatively stable and chemically unreactive, but a knowledge of the particle size of gold suspensions is necessary before they can be used in further studies and it is an advantage to use a method of preparation that provides control of their size.

A method for the preparation of monodisperse suspensions of gold particles has now been developed by Dr. G. Frens of Philips Research Laboratories, Eindhoven (Nature Phys. Sci., 1973, 241, 20-23). The size of the gold particles can be controlled in the range $150 \AA$ to $1500 \AA$. The method is based on the work of J. Turkevich et al. (Disc. Farad. Soc., 1951, 11, 55), who found that the reduction of chlorauric acid solution with sodium citrate gave a reproducible suspension of spherical gold particles within a narrow size range. The present work has shown that the number of gold nuclei formed at the start of the reduction depends upon the amount of sodium citrate used. Small amounts of citrate produce relatively few nuclei which then grow to form large particles as the residual gold in solution is reduced. Larger amounts of citrate produce more nuclei which grow to a less extent

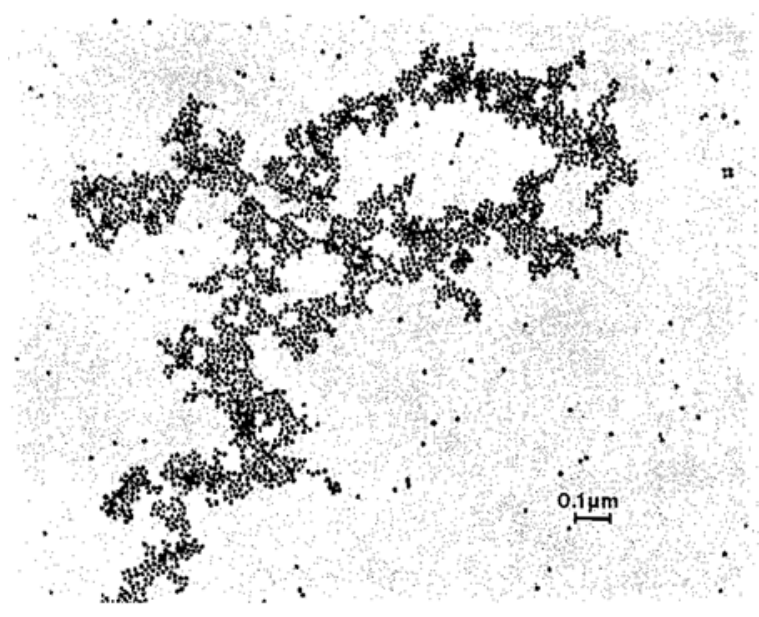

Electron micrograph of the finest gold suspension, with particle diameters of $160 \AA$ and produce small particles because the available gold is distributed over the greater number of nuclei.

Frens has shown that effect on particle size is dependent upon control over the relation between nucleation and growth and not on the amount of gold reduced from solution. $\mathrm{He}$ has done this by showing that the ratio of the number of gold particles per unit volume of suspensions of different particit size is in accordance with the same proportion of gold having been reduced in each case. The fact that no further change in the characteristics of the suspensions is caused by additions of more sodium citrate indicates that all the gold in solution is reduced. The technique of controlling the degree of nucleation is more convenient than methods that depend upon the use of a different nucleating agent before adding a reductant capable of causing growth only on existing nuclei.

Dr. Frens has used a series of suspensions of gold particles prepared by the new method to demonstrate the increased tendency of metal suspensions to coagulate in the presence of electrolytes as the radius of the particles increases. The uniformity of size and the regular spheroidal shape of the particles is shown in the electron micrographs of the smallest and largest particle size suspensions described. Similar regularity of particle characteristics is obtained when suspensions with intermediate particle sizes are produced.

o. N. C.

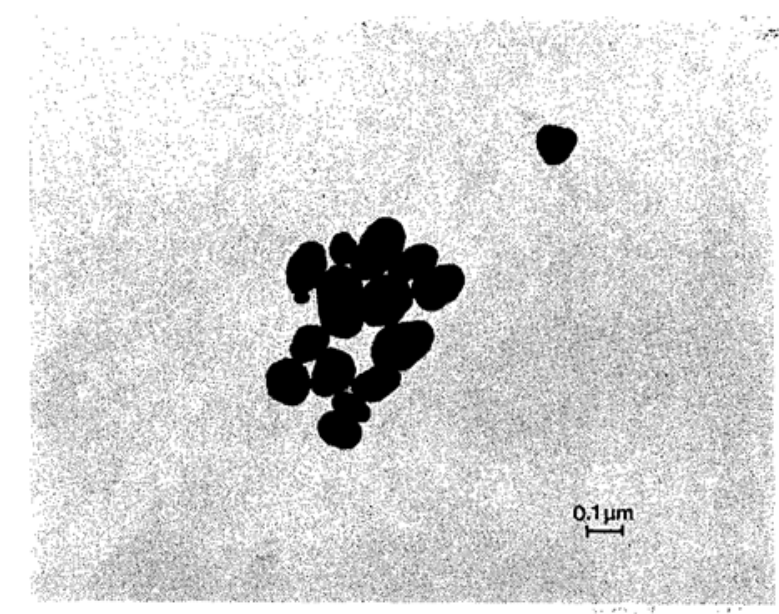

The largest of the range of six particle sizes, averaging $1500 \AA$ in diameter 